\title{
Estimated Resource Costs for Implementation of CDC's Recommended COVID-19 Mitigation Strategies in Pre-Kindergarten through Grade 12 Public Schools - United States, 2020-21 School Year
}

\author{
Ketra L. Rice, $\mathrm{PhD}^{1}$; Gabrielle F. Miller, $\mathrm{PhD}^{1}$; Fátima Coronado, $\mathrm{MD}^{1}$; Martin I. Meltzer, $\mathrm{PhD}^{1}$
}

On December 11, 2020, this report was posted as an MMWR Early Release on the MMWR website (https://www.cdc.gov/mmwr).

As school districts across the United States consider how to safely operate during the 2020-21 academic year, CDC recommends mitigation strategies that schools can adopt to reduce the risk for transmission of SARS-CoV-2, the virus that causes coronavirus disease 2019 (COVID-19) (1). To identify the resources and costs needed to implement school-based mitigation strategies and provide schools and jurisdictions with information to aid resource allocation, a microcosting methodology was employed to estimate costs in three categories: materials and consumables, additional custodial staff members, and potential additional transportation. National average estimates, using the national pre-kindergarten through grade 12 (preK-12) public enrollment of 50,685,567 students, range between a mean of $\$ 55$ (materials and consumables only) to $\$ 442$ (all three categories) per student. State-by-state estimates of additional funds needed as a percentage of fiscal year 2018 student expenditures (2) range from an additional $0.3 \%$ (materials and consumables only) to $7.1 \%$ (all three categories); however, only seven states had a maximum estimate above $4.2 \%$. These estimates, although not exhaustive, highlight the level of resources needed to ensure that schools reopen and remain open in the safest possible manner and offer administrators at schools and school districts and other decision-makers the cost information necessary to budget and prioritize school resources during the COVID-19 pandemic.

Approximately 50 million students are enrolled in public elementary and secondary schools in the United States (3); since March 2020, approximately 270,000 cases of COVID-19 have been reported among school-aged children (aged 5-17 years) (4). Although current evidence indicates that the risk for SARS-CoV-2-related hospitalizations and deaths among children is lower than that among adults, the risk for morbidity and mortality posed to teachers and other staff members in the school environment is expected to mirror that of other adults with similar demographic characteristics in the community (5). As school districts across the United States consider how to safely operate schools for the 2020-21 academic year, CDC provides indicators to help local jurisdictions determine the risk associated with operating schools for in-person learning. These indicators include measures of underlying community transmission and a measure of adherence to five primary mitigation strategies (1): 1) consistent and correct use of masks, 2) social distancing to the extent possible, 3) hand hygiene and respiratory etiquette, 4) cleaning and disinfection, and 5) contact tracing in collaboration with the local health department. Other mitigation strategies that can also be used concurrently include cohorting and staggered scheduling (1). In this analysis, the resources needed to implement four of the five key mitigation strategies were identified and costs estimated, with the goal of providing estimates to aid resource allocation to ensure the safe operation of schools and reduce school-based transmission of SARS-CoV-2. Contact tracing, although an essential strategy to reduce transmission, was excluded because those costs are not financed by school district budgets.

A microcosting approach $(\sigma)$ was used to estimate resources and costs associated with implementing the critical CDCrecommended mitigation strategies. This approach involves collecting detailed data on resources needed for each strategy and applying unit costs for those resources. From a school budget perspective, resources needed to implement the four strategies are identified (Supplementary Table, https://stacks. cdc.gov/view/cdc/97907), and total costs associated with each resource are estimated. Direct budgetary costs are the focus of this analysis; opportunity costs are excluded. The estimates indicate resources needed in addition to those already allocated in annual school budgets. Costs were aggregated and analyzed nationally and for each state and the District of Columbia (DC). A range is provided for each cost to indicate levels of cost variation around each estimate.

Personnel costs for school custodians were estimated using data from the Bureau of Labor Statistics.* To account for fringe benefits, annual wages were increased by the state and local government average of $37.8 \% .^{\dagger}$ Labor demand for school custodians was derived from a study of national standards for allocating school custodians that increases the recommended

\footnotetext{
*The Bureau of Labor Statistics (BLS) provides national and state occupational employment and wage estimates each May (in the given year); the latest published estimates are provided for May 2019. https://www.bls.gov/oes/ current/oessrcst.htm.

$\dagger$ Employer Costs for Employee Compensation reports are provided by BLS and provide benefit rates by industry type. https://www.bls.gov/bls/news-release/ ecec.htm\#2019.
} 
number of custodians from a tier 3 level of cleaning (one custodian per $28,000-31,000 \mathrm{ft}^{2}$ ) to a tier 2 level of cleaning (one custodian per 18,000-20,000 $\mathrm{ft}^{2}$ ) (7). The American Federation of Teachers estimates that tier 2 cleaning is needed for an estimated $10 \%$ of targeted physical areas per school (i.e., bathrooms, food service areas, and high-need classrooms, including special needs classes) (8). To allow for variation in school size, ranges of estimates for additional custodial services were estimated. The low estimate used an additional 1.25 full time equivalents (FTEs), and the high estimate used 2.5 FTEs (Supplementary Table, https://stacks.cdc.gov/view/cdc/97907) (7). Costs are inflated and reported in 2020 U.S. dollars. ${ }^{\$}$ Potential additional transportation costs were extrapolated from a report by the American Federation of Teachers that forecasts an estimated 36\% national increase in funding needed for school transportation ( 7 ). These potential costs assume that some schools would require additional buses, drivers, and protocols to implement social distancing on buses. The 36\% national increase was distributed across states and adjusted by states' past year transportation spending per student (8). Ranges for nonlabor costs for all materials and consumables were obtained from the U.S. General Services Administration (GSA) Supply Catalog 2020, GSA Advantage Disaster Relief and Pandemic Products online catalog, and through various e-commerce marketplace websites to derive a range of cost estimates across multiple sources and reflect price variability for materials across vendors. Aggregated material costs were adjusted for each state using the 2020 state-based composite cost of living index.** Average costs per student were calculated using the national preK-12 public student enrollment of 50,685,567 students (3). State-based cost estimates were adjusted based on the number of schools and the total school population within each state. Estimated pandemic-related per-student costs were calculated as a percentage of fiscal year 2018 per-student expenditures as reported by the National Center for Education Statistics (2).

National costs per student range between a mean of $\$ 55$ (materials and consumables only) to $\$ 442$ (three categories) (Table 1). The highest cost categories were related to employing additional custodians per school (44.8\% of total costs)

\footnotetext{
$\$$ Consumer Price Index databases are provided annually by BLS. https://www. bls.gov/cpi/data.htm.

9 Nonlabor material sources retrieved from U.S. General Services Administration (GSA) Global Supply Catalog 2020 (https://www.gsaglobalsupply.gsa.gov/ advantage/); GSA Advantage (https://www.gsaadvantage.gov/advantage) Disaster Relief and Pandemic Products Supply; School Kids Healthcare (https:// www.buyemp.com/school-kids-healthcare-transition), School Health (https:// www.schoolhealth.com), School Nurse Supply (https://www. schoolnursesupplyinc.com), and Amazon Marketplace (https://www.amazon. $\mathrm{com} / \mathrm{s}$ ? $\mathrm{k}=$ coronavirus).

** The Missouri Economic Research and Information Center Cost of Living Data Series provides state level cost of living indices for 2020. https://meric. mo.gov/data/cost-living-data-series.
}

and potential additional transportation ( $42.8 \%$ of total cost). For state-based estimates, the incremental increase in costs per student for materials and consumables ranges from $\$ 47$ to $\$ 109$ per student; implementation of all strategies combined (including high and low projections for additional custodial staff members) range between $\$ 204$ (Utah) and \$912 (DC) (Table 2). Utah's and DC's average total costs are lower and higher, respectively, than the national range (Table 1) because of their lower and higher transportation costs per pupil, relative to other states. All other state estimates fall within the national range. Additional funds needed as a percentage of fiscal year 2018 per-student expenditures range from 0.3\% (materials and consumables only) to $7.1 \%$ (all three categories), although only seven states had a maximum estimate $>4.2 \%$ (Table 2 ).

\section{Discussion}

Successfully operating schools during the COVID-19 pandemic requires sufficient resources to implement and sustain effective mitigation strategies. This cost estimate for the resources needed to safely reopen and keep schools open for in-person learning found that the average school district will need to invest $\$ 55$ per student for materials and consumables only. This cost increases to a maximum average of $\$ 442$ per student if a school district needs or chooses to employ the maximum number of additional custodial staff members per school and add additional transportation. Costs might be lower, depending on the extent of the learning model as schools transition from virtual to hybrid or in-person learning. These estimates provide schools, districts, and other jurisdictions with the cost information necessary to budget and prioritize resources during the COVID-19 pandemic.

The findings in this report are subject to at least four limitations. First, costs related to food service operations were not included. Although some schools might incur additional costs to provide student meals, estimates might significantly vary given differences in the need for school meal programs across districts. Second, a 1-month supply of face masks for the school population was estimated and was not included as an ongoing cost for schools, based on the assumption that teachers and staff members would purchase their own masks, and schools would add masks to the student supply list. Third, costs related to social distancing within the classroom were not estimated because other resources for schools recommended by CDC (e.g., physical barriers in the classroom, such as individual student desk shields) (9) were included in the estimates. Resource needs and costs for social distancing will vary with individual school needs. Finally, although contact tracing is a primary mitigation strategy, costs for contact tracing were excluded because school districts do not bear the financial responsibility for hiring and employing contact tracers. 
TABLE 1. Estimated national costs for selected resources needed for school-based implementation of CDC's recommended COVID-19 mitigation strategies - United States, 2020-21 school year

\begin{tabular}{|c|c|c|c|c|c|}
\hline Cost item & $\begin{array}{l}\text { Unit cost, } \\
\text { USD* }\end{array}$ & No. of units ${ }^{\dagger}$ & $\begin{array}{l}\text { Total cost, } \\
\text { USD }\end{array}$ & $\begin{array}{c}\text { Unit cost range, } \\
\text { USD* }\end{array}$ & $\begin{array}{c}\text { Total cost range, } \\
\text { USD }\end{array}$ \\
\hline \multicolumn{6}{|l|}{ Materials and consumables } \\
\hline Plexiglass shield (1 per school) & 74.99 & 98,456 & $7,383,215$ & $49.50-147.75$ & $4,873,572-19,272,762$ \\
\hline Student desk shields (1 per student) & 37.20 & $50,685,567$ & $1,885,503,092$ & 14.99-75.95 & $759,776,649-3,849,568,814$ \\
\hline Reusable face shield ( 1 per teacher and other staff member) & 4.88 & $6,382,813$ & $31,148,128$ & $1.93-17.40$ & $12,318,829-111,060,947$ \\
\hline Disposable face masks (1-month supply per student, teacher, and staff member) & 0.31 & $1,141,367,601$ & $353,823,956$ & $0.10-1.50$ & $114,136,760-1,712,051,402$ \\
\hline Disposable gloves ( 2 pair per teacher and other staff member) & 0.18 & $12,765,626$ & $2,297,813$ & $0.17-0.25$ & $2,170,156-3,191,407$ \\
\hline Hand sanitizer dispenser (4 units per school) & 109.89 & 393,824 & $43,277,319$ & $81.67-137.36$ & $32,163,606-54,095,665$ \\
\hline Hand sanitizer dispenser refills ( 1 refill per month per unit per school) & 2.07 & $3,938,240$ & $8,163,972$ & $1.55-2.58$ & $6,104,272-10,160,659$ \\
\hline Hand sanitizer ( 1 bottle per student) & 4.89 & $50,685,567$ & $247,852,423$ & $3.67-6.11$ & $186,016,031-309,688,814$ \\
\hline Multipurpose cleaners (180-day supply per school) & 4.48 & $17,722,080$ & $79,394,918$ & $3.36-5.60$ & $59,546,189-99,243,648$ \\
\hline Disinfectants/Virucides (180-day supply per school) & 4.99 & $17,722,080$ & $88,344,569$ & $3.74-6.24$ & $66,280,579-110,585,779$ \\
\hline No touch thermometer ( 2 per school) & 59.00 & 196,912 & $11,617,808$ & 25.99-75.99 & $5,117,743-14,963,343$ \\
\hline Oximeter ( 2 per school) & 84.99 & 196,912 & $16,735,551$ & 15.95-199.99 & $3,140,746-39,380,431$ \\
\hline $\begin{array}{l}\text { Signage ( } 1 \text { kit of } 100 \text { hallway floor signs and } 30 \text { hallway directional arrows } \\
\text { per school) }\end{array}$ & 268.44 & 98,456 & $26,429,529$ & $178.96-357.92$ & $18,604,246-35,239,372$ \\
\hline Total materials and consumables $\S$ & - & - & $2,801,972,293$ & - & $1,075,901,224-12,584,162,010$ \\
\hline \multicolumn{6}{|l|}{ Personnelף } \\
\hline Custodian FTEs (high estimate) ${ }^{* *}$ & 40,837 & 246,140 & $10,051,712,049$ & $31,314-51,953$ & $7,707,613,702-12,699,213,527$ \\
\hline Custodian FTEs (low estimate) ${ }^{* *}$ & 40,837 & 123,070 & $5,025,824,797$ & $31,314-51,953$ & $3,853,806,851-6,349,606,764$ \\
\hline Potential additional transportation ${ }^{\dagger \dagger}$ & - & - & $9,600,000,000$ & - & $8,131,200,000-18,969,600,000$ \\
\hline \multicolumn{6}{|l|}{ Cost per student ${ }^{\S \S}$} \\
\hline Average materials and consumables cost per student & - & - & 55 & - & $21-248$ \\
\hline Average personnel cost per student (high) & - & - & 198 & - & $152-251$ \\
\hline Average personnel cost per student (low) & - & - & 99 & - & $76-125$ \\
\hline Average potential transportation cost per student ${ }^{\S \S}$ & - & - & 189 & - & $160-374$ \\
\hline
\end{tabular}

Abbreviations: COVID-19 = coronavirus disease 2019; FTEs = full-time equivalents; USD = U.S. dollars.



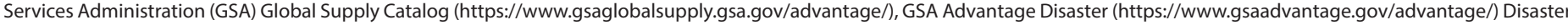

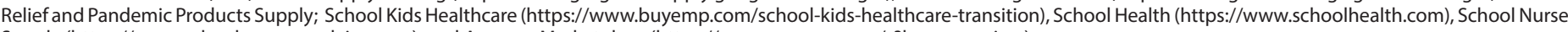
Supply (https://www.schoolnursesupplyinc.com), and Amazon Marketplace (https://www.amazon.com/s?k=coronavirus).

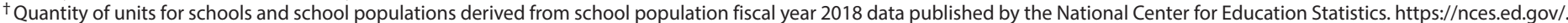

$\S$ Cost range for materials and consumables adjusted by lowest and highest state composite cost of living index. https://meric.mo.gov/data/cost-living-data-series.

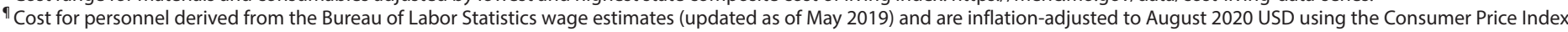
(CPI) Databases (https://www.bls.gov/cpi/data.htm); all other costs reported in current 2020 USD.

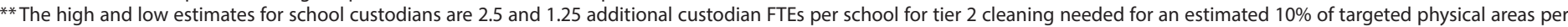

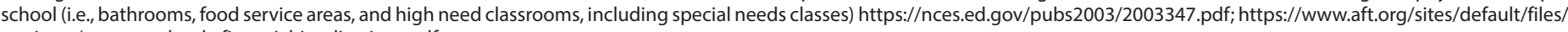
wysiwyg/reopen-schools-financial-implications.pdf.

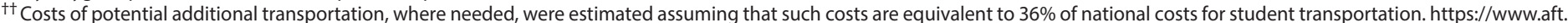
org/sites/default/files/wysiwyg/reopen-schools-financial-implications.pdf.

$\S \S$ Based on national pre-kindergarten-grade 12 public student enrollment of 50,685,567 students. https://nces.ed.gov/.

\section{Summary}

What is already known about this topic?

CDC recommends mitigation strategies that schools can adopt to minimize the risk for transmission of SARS-CoV-2 in school settings.

What is added by this report?

Costs per student for implementation of strategies range from a mean of $\$ 55$ (materials and consumables only) to $\$ 442$ (materials and consumables, additional custodial staff members, and potential additional transportation). Incremental costs across states range from an additional $0.3 \%$ to $7.1 \%$ in costs needed above reported fiscal year 2018 school expenditures per student.

What are the implications for public health practice?

These findings offer schools, school districts, and other decision makers cost information necessary to budget and prioritize school resources during the COVID-19 pandemic.
The benefits of schools extend beyond academic achievements and have critical implications for student health, safety, social and emotional well-being, and the economy, because in-person learning allows parents and caretakers to return to work (9). Although the list of resources identified in this analysis is not exhaustive, the cost estimates illustrate the level of resources needed to help ensure that schools both reopen and operate in the safest possible manner. In addition, this report provides cost data that can be used as a baseline for future studies examining the cost-effectiveness of mitigation strategies in school settings and those comparing costs and benefits across multiple sectors of the economy.

Corresponding author: Ketra Rice, wss1@cdc.gov.

${ }^{1}$ CDC COVID-19 Response Team.

All authors have completed and submitted the International Committee of Medical Journal Editors form for disclosure of potential conflicts of interest. No potential conflicts of interest were disclosed. 
TABLE 2. Estimated costs for selected resources needed for school-based implementation of CDC's recommended COVID-19 mitigation strategies, by state - United States, 2020-21 school year

\begin{tabular}{|c|c|c|c|c|c|c|c|c|c|c|c|}
\hline \multirow[b]{2}{*}{ State } & \multirow[b]{2}{*}{$\begin{array}{c}\text { No. of } \\
\text { schools* }\end{array}$} & \multirow[b]{2}{*}{$\begin{array}{l}\text { No. of } \\
\text { teachers/ } \\
\text { staff } \\
\text { members* }\end{array}$} & \multirow[b]{2}{*}{$\begin{array}{c}\text { Total } \\
\text { student } \\
\text { enrollment* }\end{array}$} & \multicolumn{7}{|c|}{ Cost, USD } & \multirow[b]{2}{*}{$\begin{array}{c}\text { Pandemic } \\
\text { costs, } \% \\
\text { increase, }^{* *} \\
\text { range }\end{array}$} \\
\hline & & & & $\begin{array}{c}\text { Materials/ } \\
\text { Consumables }\end{array}$ & 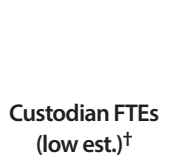 & $\begin{array}{l}\text { Custodian FTEs } \\
\text { (high est.) }^{\dagger}\end{array}$ & $\begin{array}{c}\text { Potential } \\
\text { additional } \\
\text { transportation }^{\S}\end{array}$ & $\begin{array}{l}\text { Avg. total } \\
\text { cost per } \\
\text { student, } \\
\text { range }\end{array}$ & $\begin{array}{l}\text { Avg. cost per } \\
\text { student } \\
\text { (materials/ } \\
\text { consumables } \\
\text { only) }\end{array}$ & $\begin{array}{c}\text { FY18* } \\
\text { expenditures } \\
\text { per student }\end{array}$ & \\
\hline Alabama & 1,509 & 71,628 & 742,444 & $36,773,905$ & $63,369,776$ & $126,739,552$ & $136,505,880$ & $319-404$ & 50 & 9,717 & $0.5-4.2$ \\
\hline Alaska & 508 & 16,982 & 132,872 & $9,431,191$ & $30,381,042$ & $60,762,083$ & $29,442,960$ & $521-750$ & 71 & 17,726 & $0.4-4.2$ \\
\hline Arizona & 2,284 & 103,175 & $1,110,851$ & $61,469,251$ & $111,849,022$ & $223,698,043$ & $136,946,520$ & $279-380$ & 55 & 8,373 & $0.7-4.5$ \\
\hline Arkansas & 1,088 & 73,658 & 496,085 & $24,187,597$ & $46,814,518$ & $93,629,037$ & $68,657,040$ & $282-376$ & 49 & 10,168 & $0.5-3.7$ \\
\hline California & 10,303 & 577,836 & $6,304,266$ & $497,309,250$ & $618,835,013$ & $1,237,670,026$ & $638,704,080$ & $278-377$ & 79 & 12,664 & $0.6-3.0$ \\
\hline Colorado & 1,862 & 111,939 & 910,280 & $51,930,527$ & $95,898,121$ & $191,796,241$ & $99,713,880$ & $272-377$ & 57 & 10,238 & $0.6-3.7$ \\
\hline Connecticut & 1,369 & 98,166 & 531,288 & $36,477,044$ & $84,231,421$ & $168,462,843$ & $189,668,880$ & $584-743$ & 69 & 20,147 & $0.3-3.7$ \\
\hline Delaware & 223 & 17,098 & 136,293 & $8,114,416$ & $11,223,913$ & $22,447,827$ & $37,752,480$ & $419-501$ & 60 & 15,282 & $0.4-3.3$ \\
\hline District of Columbia & 228 & 14,106 & 87,315 & $7,756,625$ & $13,773,041$ & $27,546,082$ & $44,319,600$ & $754-912$ & 89 & 23,155 & $0.4-3.9$ \\
\hline Florida & 4,322 & 345,644 & $2,832,424$ & $155,323,788$ & $197,729,771$ & $395,459,542$ & $381,070,800$ & $259-329$ & 55 & 9,663 & $0.6-3.4$ \\
\hline Georgia & 2,297 & 224,488 & $1,768,642$ & $87,993,477$ & $97,806,719$ & $195,613,439$ & $323,339,040$ & $288-343$ & 50 & 10,760 & $0.5-3.2$ \\
\hline Hawaii & 290 & 22,596 & 180,837 & $19,753,419$ & $16,819,007$ & $33,638,014$ & $23,746,680$ & $334-427$ & 109 & 15,242 & $0.7-2.8$ \\
\hline Idaho & 744 & 27,186 & 301,186 & $15,700,537$ & $33,499,456$ & $66,998,911$ & $38,018,160$ & $290-401$ & 52 & 7,846 & $0.7-5.1$ \\
\hline Illinois & 4,175 & 260,463 & $2,005,153$ & $99,427,837$ & $221,424,361$ & $442,848,721$ & $506,354,760$ & $413-523$ & 50 & 15,912 & $0.3-3.3$ \\
\hline Indiana & 1,921 & 152,826 & $1,054,187$ & $52,156,534$ & $\$ 92,649,830$ & $185,299,660$ & $234,095,040$ & $359-447$ & 49 & 10,033 & $0.5-4.5$ \\
\hline lowa & 1,349 & 72,886 & 511,850 & $25,861,692$ & $67,664,761$ & $135,329,522$ & $77,619,960$ & $334-467$ & 51 & 11,724 & $0.4-4.0$ \\
\hline Kansas & 1,320 & 73,271 & 497,088 & $23,769,356$ & $61,662,744$ & $123,325,488$ & $81,582,480$ & $336-460$ & 48 & 11,095 & $0.4-4.1$ \\
\hline Kentucky & 1,541 & 97,712 & 680,978 & $35,461,085$ & $71,535,339$ & $143,070,678$ & $148,091,760$ & $375-480$ & 52 & 11,081 & $0.5-4.3$ \\
\hline Louisiana & 1,390 & 107,600 & 715,135 & $36,963,040$ & $54,206,386$ & $108,412,772$ & $170,258,760$ & $366-441$ & 52 & 11,636 & $0.4-3.8$ \\
\hline Maine & 611 & 35,241 & 180,473 & $11,492,983$ & $33,131,047$ & $66,262,095$ & $48,734,640$ & $517-701$ & 64 & 15,069 & $0.4-4.7$ \\
\hline Maryland & 1,437 & 115,516 & 893,684 & $63,235,650$ & $72,450,055$ & $144,900,111$ & $255,236,040$ & $437-518$ & 71 & 15,155 & $0.5-3.4$ \\
\hline Massachusetts & 1,862 & 128,291 & 964,791 & $69,173,739$ & $118,317,113$ & $236,634,225$ & $282,796,200$ & $487-610$ & 72 & 18,328 & $0.4-3.3$ \\
\hline Michigan & 3,468 & 181,468 & $1,516,398$ & $75,527,660$ & $169,352,411$ & $338,704,821$ & $270,858,960$ & $340-452$ & 50 & 11,688 & $0.4-3.9$ \\
\hline Minnesota & 2,478 & 117,236 & 884,944 & $49,506,741$ & $140,130,095$ & $280,260,189$ & $237,747,960$ & $483-641$ & 56 & 12,910 & $0.4-5.0$ \\
\hline Mississippi & 1,076 & 67,757 & 478,321 & $22,396,022$ & $41,497,850$ & $82,995,700$ & $75,434,040$ & $291-378$ & 47 & 8,909 & $0.5-4.2$ \\
\hline Missouri & 2,424 & 128,938 & 915,472 & $44,686,239$ & $117,494,068$ & $234,988,135$ & $191,587,680$ & $386-515$ & 49 & 11,034 & $0.4-4.7$ \\
\hline Montana & 823 & 21,329 & 149,474 & $8,940,482$ & $41,734,659$ & $83,469,318$ & $29,641,320$ & $537-817$ & 60 & 11,512 & $0.5-7.1$ \\
\hline Nebraska & 1,085 & 47,292 & 323,766 & $16,609,144$ & $53,805,991$ & $107,611,982$ & $43,724,880$ & $353-519$ & 51 & 12,813 & $0.4-4.0$ \\
\hline Nevada & 662 & 26,430 & 485,785 & $29,217,364$ & $36,329,799$ & $72,659,597$ & $61,859,880$ & $262-337$ & 60 & 9,040 & $0.7-3.7$ \\
\hline New Hampshire & 490 & 31,981 & 179,433 & $10,712,581$ & $25,540,197$ & $51,080,393$ & $48,024,360$ & $470-612$ & 60 & 16,588 & $0.4-3.7$ \\
\hline New Jersey & 2,588 & 236,559 & $1,408,102$ & $95,276,011$ & $145,057,788$ & $290,115,576$ & $434,831,040$ & $479-583$ & 68 & 20,316 & $0.3-2.9$ \\
\hline New Mexico & 884 & 37,573 & 334,345 & $16,116,820$ & $38,021,569$ & $76,043,139$ & $38,696,040$ & $278-391$ & 48 & 9,963 & $0.5-3.9$ \\
\hline New York & 4,824 & 372,692 & $2,724,663$ & $234,815,599$ & $302,459,976$ & $604,919,952$ & $1,042,712,640$ & $580-691$ & 86 & 23,686 & $0.4-2.9$ \\
\hline North Carolina & 2,603 & 190,855 & $1,553,513$ & $82,013,680$ & $111,732,994$ & $223,465,988$ & $218,620,080$ & $265-337$ & 53 & 9,277 & $0.6-3.6$ \\
\hline North Dakota & 518 & 17,984 & 111,920 & $5,970,395$ & $28,284,484$ & $56,568,967$ & $22,965,480$ & $511-764$ & 53 & 13,783 & $0.4-5.5$ \\
\hline Ohio & 3,619 & 322,611 & $1,704,399$ & $86,775,862$ & $179,032,654$ & $358,065,308$ & $381,186,000$ & $380-485$ & 51 & 12,893 & $0.4-3.8$ \\
\hline Oklahoma & 1,800 & 85,914 & 695,092 & $33,314,203$ & $76,148,280$ & $152,296,560$ & $64,328,040$ & $250-360$ & 48 & 8,174 & $0.6-4.4$ \\
\hline Oregon & 1,242 & 65,928 & 608,014 & $45,139,592$ & $67,047,072$ & $134,094,145$ & $112,797,000$ & $370-480$ & 74 & 11,903 & $0.6-4.0$ \\
\hline Pennsylvania & 3,019 & 241,548 & $1,726,809$ & $98,512,658$ & $154,654,766$ & $309,309,532$ & $486,006,840$ & $428-518$ & 57 & 16,377 & $0.3-3.2$ \\
\hline Rhode Island & 313 & 19,482 & 142,949 & $9,372,034$ & $16,918,292$ & $33,836,583$ & $38,119,680$ & $451-569$ & 66 & 16,954 & $0.4-3.4$ \\
\hline South Carolina & 1,248 & 78,108 & 777,507 & $41,046,461$ & $51,721,301$ & $103,442,602$ & $116,117,640$ & $269-335$ & 53 & 10,705 & $0.5-3.1$ \\
\hline South Dakota & 698 & 19,543 & 137,823 & $7,595,999$ & $32,185,705$ & $64,371,410$ & $18,681,120$ & $424-658$ & 55 & 10,263 & $0.5-6.4$ \\
\hline Tennessee & 1,859 & 128,469 & $1,001,967$ & $49,849,862$ & $79,092,549$ & $158,185,099$ & $130,509,000$ & 259-338 & 50 & 9,599 & $0.5-3.5$ \\
\hline Texas & 8,826 & 690,078 & $5,401,341$ & $273,803,482$ & $391,623,742$ & $783,247,483$ & $564,675,120$ & $228-300$ & 51 & 9,670 & $0.5-3.1$ \\
\hline Utah & 1,033 & 56,146 & 668,274 & $35,796,979$ & $45,622,342$ & $91,244,683$ & $54,689,400$ & $204-272$ & 54 & 7,576 & $0.7-3.6$ \\
\hline Vermont & 314 & 18,183 & 88,028 & $5,678,841$ & $17,751,189$ & $35,502,379$ & $21,240,720$ & $507-709$ & 65 & 20,149 & $0.3-3.5$ \\
\hline Virginia & 2,133 & 178,550 & $1,291,462$ & $72,391,508$ & $99,604,648$ & $199,209,295$ & $297,275,760$ & $363-440$ & 56 & 12,224 & $0.5-3.6$ \\
\hline Washington & 2,427 & 94,882 & $1,110,367$ & $68,869,580$ & $153,215,600$ & $306,431,200$ & $205,493,040$ & $385-523$ & 62 & 12,985 & $0.5-4.0$ \\
\hline West Virginia & 744 & 38,452 & 272,266 & $13,906,999$ & $34,614,395$ & $69,228,791$ & $86,911,560$ & $497-625$ & 51 & 11,572 & $0.4-5.4$ \\
\hline Wisconsin & 2,255 & 101,250 & 860,753 & $45,536,382$ & $112,992,469$ & $225,984,938$ & $162,415,440$ & $373-504$ & 53 & 12,446 & $0.4-4.1$ \\
\hline Wyoming & 370 & 17,268 & 94,258 & $4,924,000$ & $19,483,025$ & $38,966,051$ & $26,206,560$ & $537-744$ & 52 & 16,134 & $0.3-4.6$ \\
\hline Total costs ${ }^{t \dagger}$ & - & - & - & $3,014,066,119$ & $4,998,422,363$ & $9,996,844,725$ & $9,436,012,920$ & - & - & - & - \\
\hline
\end{tabular}

Abbreviations: avg. = average; COVID-19 = coronavirus disease 2019; est. = estimate; FTEs = full-time equivalents; FY = fiscal year; USD = U.S. dollars.

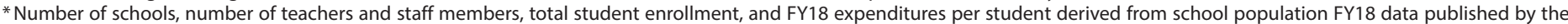
National Center for Education Statistics. https://nces.ed.gov/.

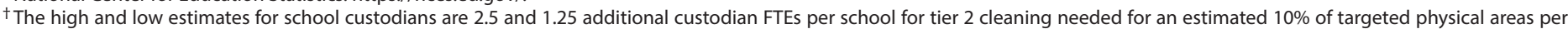

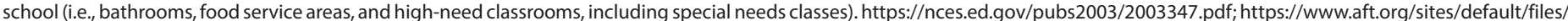
wysiwyg/reopen-schools-financial-implications.pdf.

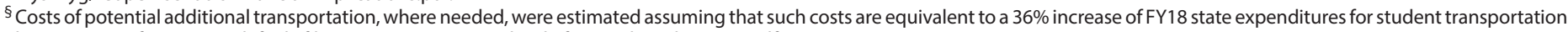
https://www.aft.org/sites/default/files/wysiwyg/reopen-schools-financial-implications.pdf.

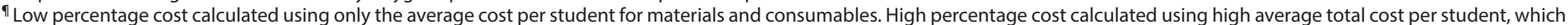
includes all three cost categories (Materials and Consumables, Custodian FTEs [high est.], and Potential additional transportation).

** Percentage increase in expenditure per student above FY18 levels.

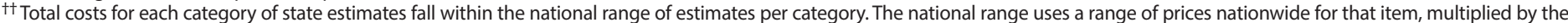

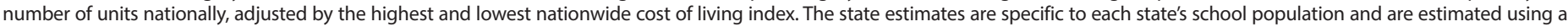

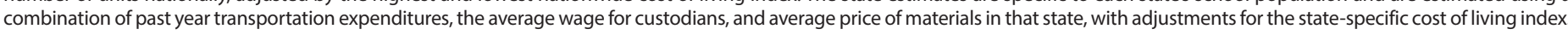




\section{References}

1. CDC. Coronavirus disease 2019 (COVID-19) indicators for dynamic school decision-making. Atlanta, GA: US Department of Health and Human Services, CDC; 2020. https://www.cdc.gov/coronavirus/2019ncov/community/schools-childcare/indicators.html

2. National Center for Education Statistics. Revenues and expenditures for public elementary and secondary school districts: FY 18. Washington, DC: US Department of Education, National Center for Education Statistics; 2020. https://nces.ed.gov/pubsearch/pubsinfo.asp?pubid=2020308

3. Hussar B, Zhang J, Hein S, et al. The condition of education 2020. NCES 2020-144. Washington, DC: U.S. Department of Education, National Center for Education Statistics; 2020. https://nces.ed.gov/ pubs2020/2020144.pdf

4. Leeb RT, Price S, Sliwa S, et al. COVID-19 trends among school-aged children-United States, March 1-September 19, 2020. MMWR Morb Mortal Wkly Rep 2020;69:1410-5. PMID:33001869 https://doi. org/10.15585/mmwr.mm6939e2
5. Selden TM, Berdahl TA, Fang Z. The risk of severe COVID-19 within households of school employees and school-age children. Health Aff (Millwood) 2020;39:2002-9. PMID:32941086 https://doi.org/10.1377/ hlthaff.2020.01536

6. Xu X, Grossetta Nardini HK, Ruger JP. Micro-costing studies in the health and medical literature: protocol for a systematic review. Syst Rev 2014;3:47. PMID:24887208 https://doi.org/10.1186/2046-4053-3-47

7. National Forum on Education Statistics. Planning guide for maintaining school facilities. NCES 2003-347. Washington, DC: US Department of Education, National Center for Education Statistics, National Forum on Education Statistics; 2003. https://nces.ed.gov/pubs2003/2003347.pdf

8. American Federation of Teachers. Reopening schools during a time of triple crisis: financial implications. Washington, DC: American Federation of Teachers; 2020. https://www.aft.org/sites/default/files/wysiwyg/reopenschools-financial-implications.pdf

9. CDC. Coronavirus disease 2019 (COVID-19). Operating schools during COVID-19: CDC's considerations. Atlanta, GA: US Department of Health and Human Services, CDC; 2020. https://www.cdc.gov/ coronavirus/2019-ncov/community/schools-childcare/schools.html 